\title{
A Study on Comparitive Role of Ultrasonogram and Computed Tomography in Diagnosis of Acute Pancreatitis
}

\author{
Ritesh Kondeti ${ }^{1}$, Prashanth Thimme Gowda ${ }^{2}$ \\ ${ }^{1}$ Associate Professor, ${ }^{2}$ Associate Professor, Department of Radiology, Pondicherry Institute of Medical Sciences, Pondicherry, \\ India
}

Corresponding author: Dr. Prashanth Thimme Gowda, Associate Professor, Department of Radiology, Narayana Medical College, Chinthareddypalem, Nellore, Andhra Pradesh, India

DOI: 10.21276/ijcmsr.2018.3.3.36

How to cite this article: Ritesh Kondeti, Prashanth Thimme Gowda. A study on comparitive role of ultrasonogram and computed tomography in diagnosis of acute pancreatitis. International Journal of Contemporary Medicine Surgery and Radiology. 2018;3(3):C164-C167.

\section{A B S T R A C T}

Introduction: Acute pancreatitis is an acute inflammatory disease of the pancreas characterized by auto digestion of pancreatic parenchyma, resulting from the inappropriate intracellular activity of proteolytic pancreatic enzymes. Radiological imaging is crucial in making a decision in management of patients with acute pancreatitis. The present study was aimed to study the role of ultrasonography and computed tomography in various cases of acute pancreatitis and to note the advantages of one imaging modality over the other.

Material and Methods: A two year prospective study was conducted at a tertiary care hospital after approval from ethical committee. Cases of acute pancreatitis were enrolled and socio demographic data, clinical history were noted and Ultrasonogram and Computed tomography was performed. Plain radiographs, biochemical investigations were correlated with the USG and CT findings. The collected data was entered in a Microsoft excel spread sheet and analyzed. Mean, median was calculated for constant variables.

Results: 100 cases were enrolled in study with 82 males and 18 females. 31-40 years was the common age group and the mean age of cases was $36.12 \pm 1.8$ years. Alcoholism was commonest etiology (68\%) and epigastric pain was the common symptom. $82 \%$ of cases was diagnosed by ultra sonogram whereas $100 \%$ of cases were diagnosed by CT. 18 cases were not diagnosed on USG due to bowel inflation. 52 of the cases (63.4\%) demonstrated heterogeneous hypoechoic echotexture with areas of calcification in 24 cases and 12 cases (14.63\%) homogenous hypoechoic echotexture.

Conclusion: To conclude, the overall visualization of the pancreas was far better by CT than by ultrasound. Alterations in size were better appreciated on CT. Ultrasound proved more useful in detecting free fluid. The positive predictive value of both ultrasound and CT was $100 \%$. This means that all patients with a bulky, hypoechoic pancreas on ultrasound have acute pancreatitis. Our study suggest that, a negative ultrasound study does not exclude significant and, at times, life-threatening pancreatic disease.

Keywords: Acute Pancreatitis, Ultrasonogram, Computed Tomography, Epigastric Pain.

\section{INTRODUCTION}

Acute pancreatitis is an acute inflammatory disease of the pancreas characterized by auto digestion of pancreatic parenchyma, interstitial fat necrosis, and necrotizing vasculitis resulting from the inappropriate intracellular activity of proteolytic pancreatic enzymes. At Atlanta Symposium in 1992, it was classified into two groups, as "mild" and "Severe" based on clinical and biochemical findings. ${ }^{1}$ However this was revised in 2008, by Acute Pancreatitis Classification working Group, morphologically based on imaging findings as "Interstitial oedematous pancreatitis" and "Necrotizing Pancreatitis". Hence after the classification based on imaging, pancreatic imaging requires not only for sub typing the disease but also to be familiar with appropriate nomenclature. ${ }^{2}$ Radiological imaging is crucial in making a decision in management of patients with acute pancreatitis.
Imaging choices available in diagnosing acute pancreatitis include USG, computed tomography (CT), Magnetic Resonance imaging (MRI), MRCP and ERCP. The choice depends upon the reason for investigation. Imaging of pancreas is recommended in cases of acute pancreatitis, to confirm the diagnosis, grade the extent and severity of disease. However the common complaint in majority of cases is pain abdomen where Ultrasound is the primary imaging modality which helps in evaluating the exact cause and ruling out other causes of pain abdomen. The limitation of USG is only $60-7-\%$ of visualization of pancreas is possible. Computed tomography (CT) has been a choice in cases where it crucial to evaluate the severity and to delineate pancreatic and extra pancreatic complications such as peripancraetic fluid collection, pseudocyst and pancreatic abscess. The role of CT in assessing prognosis has been investigated, by correlating the presence and extent of peripancreatic fluid collection 
with severity of the disease. Magnetic resonance imaging has gained importance in imaging teh cases of pancreatitis with Iodine allergies, in characterizing collections and assessment of an abnormal or disconnected pancreatic duct. ${ }^{3}$

The present study was aimed to study the role of ultrasonography and computed tomography in various cases of acute pancreatitis and to note the advantages of one imaging modality over the other and vis-à-vis in the diagnosis of acute pancreatitis.

\section{MATERIAL AND METHODS}

A prospective observational study was conducted for a period of two years from March 2016 to February 2018 at a tertiary care hospital. All the cases suspicious or confirmed with biochemical investigations with acute pancreatitis referred from department of Emergency medicine and Gastroenterology were included in the clinical study. The study was approved by the institutional ethical committee and study protocol was followed as per the guidelines of the committee. The socio demographic data, clinical history, signs and symptoms of the cases were noted in a separate performa and written consent was obtained from all the cases in the study. Patients were imaged using ultrasound and CT scan modalities and the findings of each case were noted in the report. Plain radiographs, biochemical investigations were correlated with the USG and CT findings.

Ultrasonography: The sonographic study was performed by Philip Envisor Doppler machine by using a linear 3-12MHz probe and a curvilinear probe. Pancreas was visualized by transverse scans in midline below the xiphoid process using the related vascular marks. Using left kidney as an acoustic window the tail of the pancreas was visualized anterior to its pole. Colour Doppler was done to visualize the vascularity of pancreas, pancreatic lesion, and associated vascular structures. Size, shape, contour and echo-texture of pancreas and the lesion area were noted. Findings of individual cases were recorded in as separate sheet.

\section{Computed tomography}

Patients who underwent USG were subjected to tomographic evaluation by using Siemens Single Slice Spiral slice CT scanner. All the cases were administered oral contrast for opacification of bowel and duodenum. Plain sections were taken off the pancreatic area. Intravenous non-ionic contrast was administered based on the age and weight and renal status. Scan slices were taken as $10 \mathrm{~mm}$ contiguous sections (pitch-1.5) over the entire upper abdomen and data reconstructed with $3 \mathrm{~mm}$ thin slices. Multi-plannar reconstructions were done when required. Axial sections were studied and noted about the size, shape, density, enhancing pattern of pancreas and pancreatic lesion, fat planes and vascular complications etc.

\section{STATISTICAL ANALYSIS}

The collected data was entered in a Microsoft excel spread sheet and analyzed. Mean, median was calculated for constant variables.

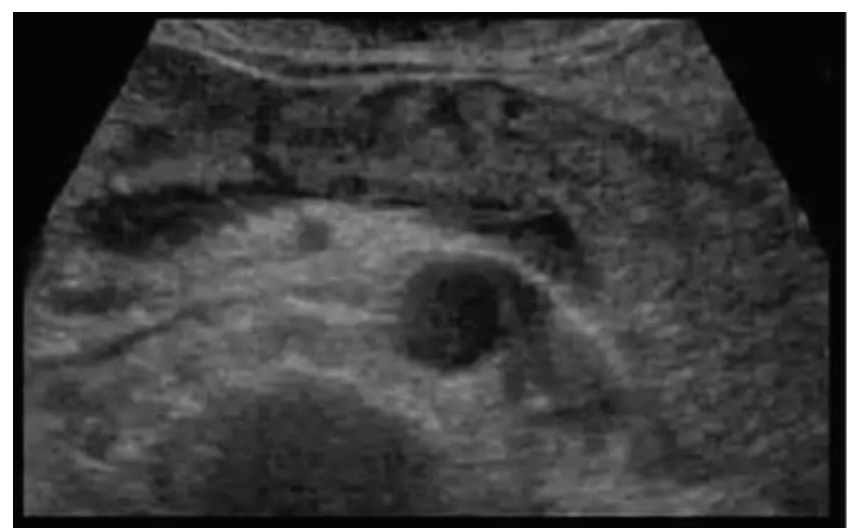

Figure-1: Ultrasonogram showing enlarged oedematous Pancreas.

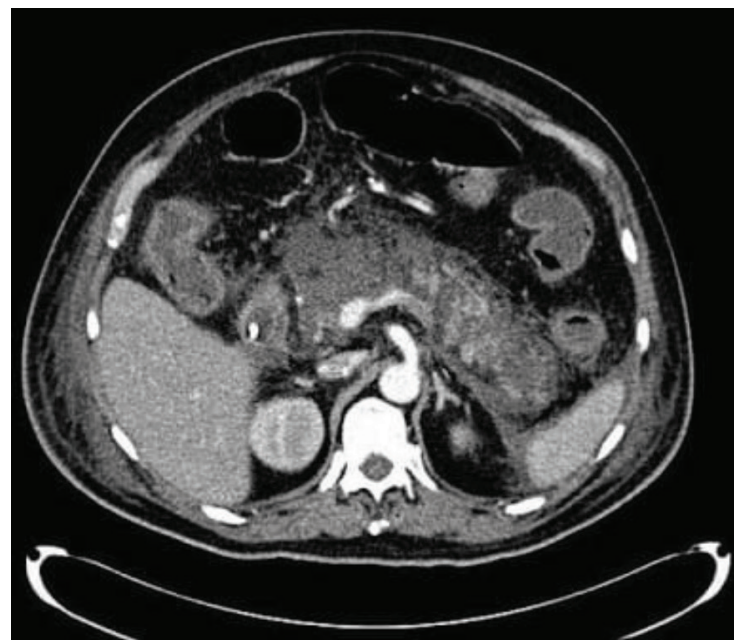

Figure-2: CECT showing diffusely enlarged Pancreas with non enhancing areas in Head, Neck suggestive of Necrosis.

\section{RESULTS}

In the present study conducted at a tertiary care hospital, a total of 100 cases of clinically suspicious and biochemically confirmed cases of acute pancreatitis were included. As a protocol, serum amylase levels were evaluated biochemically in every case and in all the 100 cases who participated, the levels were elevated more than the normal. (>200U/L) Males were predominant in the study with 82 cases $(82 \%)$ and females only 18 cases (18\%). The age of the cases in the study ranged from $14-78$ years with mean age of $36.12 \pm 1.8$ years. Maximum cases were in the age group of 31-40 years (38\%) followed by $51-60$ years (22\%), 41-50 years (20\%), 61-70 years $(8 \%),>70$ years and $<30$ years $(6 \%)$ each individually. The mean age of the males in the study was $34.28 \pm 4.8$ years and females $32.14 \pm 2.6$ years in the study. The most common aetiology in our study was Alcoholism observed in $68 \%$ of cases followed by gallstones (22\%), idiopathic (18\%), Hyperlipidemia (6\%), Trauma (2\%) and autoimmune conditions in $4 \%$ of cases, few of the cases exhibited multiple etiology. Pain abdomen (Epigastric) was the commonest symptom in all the cases with radiation of the pain to the back. Nausea and vomiting were the next common symptoms. Bilious vomiting was observed in 28 cases, weight loss in 18 cases and jaundice in 12 cases were observed. Many of the 
cases presented with multiple symptoms. Abdominal lump was found in 16 cases in the study. Serum amylase level was elevated in all the cases with 24 cases $>200 U / 1$ and 76 cases $>140 \mathrm{U} / 1$ and $<200 \mathrm{U} / 1$.

USG Observations: In the present study, USG could detect 82 cases (82\%) and was not able to visualize in 18 cases. In 12 cases the whole pancreas was masked by inflating bowel due to gas and in 6 cases the tail region could not be visualized. These 18 cases however were detected by CT. Enlarged pancreas was observed in 68 cases which may be due to pancreatic inflammation or other aetiology (malignancy etc). Diffuse involvement of pancreas was observed in 43 cases (52.44\%) and focal involvement with involvement of head, neck/body or tail was observed in 39 cases (47.56\%). Head was the major portion of the pancreatic involvement in focal cases. The contour of the pancreas was regular in 24 cases (29.26\%) and irregular in 58 cases (70.74\%). 52 of the cases (63.4\%) demonstrated heterogeneous hypoechoic echotexture with areas of calcification in 24 cases and 12 cases (14.63\%) homogenous hypoechoic echotexture. [Figure-1] Normal echotexture was identified in 18 cases (21.9\%). Calcification was observed in 12 cases (14.63\%). Dilatation of pancreatic duct $(>3 \mathrm{~mm})$ was identified in 8 cases. $(9.7 \%)$ Collection of fluid was observed in 24 cases $(29.26 \%)$ with intra fluid collection in 8 cases and peripancreatic fluid collection in 16 cases. Pancreatic pseudocysts were identified in 6 cases in our study. Gallstones were observed in 22 cases in our study. Associated observations in our study were ascites in 6 cases, pleural effusion (Unilateral- 4 cases) and Bilateral in 8 cases and biliary duct dilatation in 12 cases. Fatty liver was identified in 22 cases.

Observations on computed Tomography: Pancreatic lesions were identified in all 100 cases in CT with 98 cases of acute and 2 cases of chronic pancreatitis. Enlarged pancreas was identified in 82 cases and 4 cases of contracted pancreas (two were cases of chronic pancreatitis and two were acute on chronic pancreatitis) and normal in size in 14 cases. On CT, enlargement was due to inflammation in 68 cases (83\%) and 14 due to malignancy (17.07\%). Diffuse involvement was identified in 52 cases (52\%) by CT and focal involvement in 39 cases with involvement of head in 21 cases, followed by neck -11 cases and least in tail region -7 cases. Ill defined outline of the pancreas was identified in 24 cases and well defined margins in 14 cases. Homogenously or heterogeneously hypodense lesions were observed on CT in our study. [Figure-2] 28 cases were homodense and 26 were heterogeneously hyperdense of which, 8 cases showed cystic component. Pseudocysts were seen in 7 cases in our study. Calcification was observed in 32 cases and CT was more sensitive than USG in detection of cases with calcification. Caliber of the main pancreatic duct was normal in 20 cases and dilatation $(>3 \mathrm{~mm})$ was observed in 14 cases, CT was accurate and more sensitive in detection of cases with pancreatic duct dilatation. Fluid collections were seen in 32 cases with predominantly in the lesser sac. Stomach wall thickening was observed in 28 cases and Gerota's fascia thickening usually on the left seen in 18 cases. Free intraperitoneal fluid representing pancreatic ascites was observed in 12 cases, pleural effusion in 12 cases and other findings were biliary duct dilatation in 12 cases and Fatty liver in 22 cases. Enlarged regional lymph nodes were identified in 18 cases in our study.

\section{DISCUSSION}

Acute pancreatitis is a life threatening condition with severe morbidity and mortality if untreated and improperly managed. The rate of mortality varies from $<1 \%$ to $>20 \%$ depending upon the type of pancreatitis and also the age factor and aetiology of the condition. Arriving at an accurate diagnosis and early identification of complications are of immense help in reducing the mortality. Imaging the acute pancreatitis provides exact details of complications and type of pancreatitis thereby guiding in accurate diagnosis and management. Ultrasonography was the imaging modality of choice in initial phase of imaging studies but with limitations it was supplemented with CT for precision in localization of lesions, nature of the lesion like inflammation or necrosis.

In the present study, it is observed that the incidence of acute pancreatitis was higher in males than females as observed universally, but gall stone as a cause of pancreatitis was higher in females than males which were observed in our study and alcoholism was a common factor of causation among males in our study. The most common age group in our study was $31-40$ years with a median age of $36.12 \pm 1.8$ years similar to the study of Banks PA et al. ${ }^{4}$ As mentioned in many studies globally alcoholism was the commonest etiological factor in our study also but gallstones as a cause of pancreatitis was common in females in our study which is accepted universally. Epigastric pain was the commonest symptom in our study followed by nausea and vomiting which is on par with the findings of Robert J Lesniak. ${ }^{5}$ Serum amylase levels were evaluated in all the cases which are similar finding in universal studies. The sensitivity of USG in detection of acute pancreatitis was $82 \%$ in our study which is very high and is also observed in the study of Kamisawa $\mathrm{T}$ et $\mathrm{al}^{6}$ who reported the sensitivity as high as $84 \%$ in his study but contrary to the findings of Buscail L who reported the sensitivity as $65 \%$ in their study. ${ }^{7}$ Calleja and JS Barkin which stated that in acute pancreatitis, overlying bowel gas disturbances may obscure the pancreas in $40 \%$ of patients. ${ }^{8}$ However in the present study the rate of non detection due to gas or bowel obstruction was $12 \%$ which was very less compared to the previous study. In the present study, Enlarged pancreas was observed in 68 cases which is similar to the findings of Gandolfi $\mathrm{L}$ but contrary to the findings of Asayama $Y$ who reported the incidence as only $32 \%$ in his study. ${ }^{9,10}$ In the present study, 52 of the cases (63.4\%) demonstrated heterogeneous hypoechoic echotexture with areas of calcification in 24 cases and 12 cases (14.63\%) homogenous hypoechoic echotexture. Calcification was observed in 12 cases (14.63\%) which were similar to the findings of Balthazar EJ. ${ }^{11}$ Eight cases presented with Pancreatic duct dilatation (9.7\%) and 24 cases with fluid collection. These findings were similar to the findings of Johnson CD. ${ }^{12}$ Other less common associated findings observed in our study were Pancreatic pseudo cysts, Ascites, gallstones, pleural effusion and fatty liver.

CT visualization of the pancreas was possible in all cases 
(25 patients) due to non-interference by the overlying bowel gas. In the present study, enlarged pancreas was identified in 82 cases and 4 cases of contracted pancreas (two were cases of chronic pancreatitis and two were acute on chronic pancreatitis) were identified, findings of our study were on par with the findings of King NK et al. ${ }^{13}$ Due to lack of surgical correlation, the incidence of normal CT scans in mild acute pancreatitis is not known. On CT, enlargement was due to inflammation in 68 cases $(83 \%)$ and 14 due to malignancy $(17.07 \%)$ in the present study, this was contrary to the findings of Shyu JY who reported that malignancy was more common than inflammation in his study. ${ }^{14}$ Diffuse involvement was identified in 52 cases $(52 \%)$ by CT and focal involvement in 39 cases which was clearly defined by CT than USG in the study which is similar to reports of Yie $\mathrm{M}$ in his study. ${ }^{15}$ Homogenously or heterogeneously hypodense lesions were observed on CT in our study. 28 cases were homodense and 26 were heterogeneously hyperdense of which, 8 cases showed cystic component similar to many reports globally. Calcification was observed in 32 cases and CT was more sensitive than USG in detection of cases with calcification. Dilatation of pancreatic duct was observed in 14 cases and in our study it was found CT was more sensitive and accurate in detection of dilatation than USG; similar finding was also reported by Shrama V et al in their study. ${ }^{16}$ Stomach wall thickening was observed in 28 cases and Gerota's fascia thickening usually on the left seen in 18 cases.

\section{CONCLUSION}

To conclude, the overall visualization of the pancreas was far better by CT than by ultrasound. Alterations in size were better appreciated on CT. Ultrasound proved more useful in detecting free fluid. The positive predictive value of both ultrasound and CT was $100 \%$. This means that all patients with a bulky, hypoechoic pancreas on ultrasound have acute pancreatitis. Our study suggest that, a negative ultrasound study does not exclude significant and, at times, life-threatening pancreatic disease. Thus it is seen that both Ultrasonography and CT have roles to play in the diagnosis of pancreatitis and both are complementary to each other.

\section{REFERENCES}

1. Stear ML. Recent insights into the etiology and pathogenesis of acute biliary pancreatits. AJR 1995; 164(2):811-14.

2. Acute Pancreatitis Classification Working Gro85up. Revisionof the Atlanta classification of acute pancreatitis; 2010[Homepage on the Internet]. Available from: http://www.pancreasclub.com/resources/Atlanta Classification. (9-4-2008).

3. Balthazar EJ, Freeny PC, Van Sonnenberg E. Imaging and intervention in

4. acute pancreatitis. Radiology 1994; 193(3):297-306.

5. Banks PA. Practice guidelines in acute pancreatitis. Am J Gas-troenterol 1997;92(5):377—86.

6. Robert J Lesniak, Mark D Howen Walter, Andrew J Taylor.Spectrum of causes of pancreatic calcifications. AJR.2002;178(3):79-86.

7. Kamisawa T, Egawa N, Nakajima H, Tsuruta K,
Okamoto A, Kamata N, et al. Comparison of radiological and histological findings in autoimmune pancreatitis. Hepatogastroenterology. 2006;53(72):953-6.

8. Buscail L, Escorrou J, Moreau J, Delvaux M, Louvel D, Lapeyre F, Tregant P, Frexinos J.Endoscopic ultrasonography in chronic pancreatitis: a comparative prospective study with conventional ultrasonography, computed tomography and ERCP. Pancreas.1995. 10(1):251-257.

9. Calleja G.A., J.S Barkiaet al. Aate Pancreatitis. Medical Clin North Am 1993; 77 (5): 1037-1055.

10. Gandolfi L, Torresan F, Solmi L, Puccetti A The role of ultrasound in biliary and pancreatic disease. Eur J Ultrasound. 2003;16(4):141-159.

11. Asayama Y, Fang W, Stolpen A and Kuehn D. Detectability of pancreas divisum in patients with acute pancreatitis on multi-detector row computed tomography. Emerg Radiol 2012;19(2): 121-125.

12. Balthazar EJ. Acute pancreatitis: assessment of severity with clinical and CT evaluation. Radiology 2002;223(3): 603-613.

13. Johnson CD, Besselink $M G$ and Carter R. Acute pancreatitis. BMJ 2014;349(3):4859.

14. King NK, Powell JJ, Redhead D and Siriwardena AK. A simplified method for computed tomographic estimation of prognosis in acute pancreatitis. Scand J Gastroenterol 2003: 38(4);433-436.

15. Shyu JY, Sainani NI, Sahni VA, Chick JF, Chauhan NR, Conwell DL, et al. Necrotizing pancreatitis: diagnosis, imaging, and intervention. Radiographics 2014;34(5): 1218-1239.

16. Yie M, Jang KM, Kim MJ, Lee $Y$ and Choi D. Diagnostic value of CT features of the gallbladder in the prediction of gallstone pancreatitis. Eur J Radiol 2011; 80(2): 208-212.

17. Sharma V, Rana SS, Sharma RK, Kang M, Gupta $\mathrm{R}$ and Bhasin DK. A study of radiological scoring system evaluating extrapancreatic inflammation with conventional radiological and clinical scores in predicting outcomes in acute pancreatitis. Ann Gastroenterol 2015;28(3): 399-404.

\section{Source of Support: Nil; Conflict of Interest: None}

Submitted: 17-09-2018; Accepted: 27-09-2018; Published online: 05-10-2018 